\title{
STANDARD INELASTIC SHOCKS \\ AND THE DYNAMICS OF UNILATERAL CONSTRAINTS
}

\author{
J.J. Moreau \\ Institut de Mathématiques \\ Université des Sciences et Techniques du Languedoc \\ Montpellier
}

1. INTRODUCTION

This paper is devoted to mechanical systems with a finite number of degrees of freedom; let $q^{l}, \ldots, q^{n}$ denote (possibly local) coordinates in the configuration manifold $Q$. In addition to the constraints, bilateral and frictionless, which have permitted such a finite-dimensional parametrization of 2 , we assume the system submitted to a finite fami1y of unilateral constraints whose geometrical effect is expressed by $v$ inequalities

$$
\mathrm{f}_{\alpha}(\mathrm{q}) \leqslant 0
$$


defining a closed region $L$ of $Q$. As every greek index in the seque 1 , $\alpha$ takes its values in the set $\{1,2, \ldots, \nu\}$. The $\nu$ functions $f_{\alpha}$ are supposed $\mathrm{C}^{1}$, with nonzero gradients, at least in some neighborhood of the respective surfaces $f_{\alpha}=0 ;$ for the sake of simplicity, we assume them independent of time.

The typical instance of such a setting is provided by a system of perfectly rigid bodies which may enter into contact, and detach from each other, but can never interpenetrate. In view of this example, we shall refer to a configuration in which equality $\mathrm{f}_{\alpha}=0$ holds, by saying that contact $\alpha$ takes place. But the formalism applies as well to the unilateral constraints realized by means of inextensible strings ; equality $\mathrm{f}_{\alpha}=0$ in that case expresses that the corresponding string is taut.

Kinematically, for every motion $t \rightarrow q(t)$, the right-velocity $\dot{q}^{+}$, if it exists at the considered instant, is an element of the ndimensional linear space $E(q)$, the tangent space to 2 at the point $q(t)$; its components in this space are $\dot{q}^{i+}$, the right-derivatives of the real functions $t \rightarrow q^{i}(t)$. If the moving point $q(t)$ remains in $L$ for every $t$ and if the contact $f_{\alpha}=0$ takes place at the considered instant, one immediately finds

$$
\sum_{i} \dot{q}^{i+} \frac{\partial f_{\alpha}}{\partial q} \leqslant 0 .
$$

Therefore, if we put, for every $q \in L$,

$$
J(q)=\left\{\alpha \in\{1, \ldots, \nu\}: f_{\alpha}(q)=0\right\}
$$


the right-velocity vector necessarily belongs to the convex polyhedral cone defined in $E(q)$ as

$$
\mathrm{V}(\mathrm{q})=\left\{\mathrm{v} \in \mathrm{E}(\mathrm{q}): \forall \alpha \in \mathrm{J}(\mathrm{q}), \sum_{\mathrm{i}} \mathrm{v}^{\mathrm{i}} \frac{\partial \mathrm{f}_{\alpha}}{\partial \mathrm{q}^{\mathrm{i}}} \leqslant 0\right\} \quad ;
$$

in particular, this is the whole of $E(q)$ if $J(q)=\phi$. This set is usually called the tangent cone at the point $q$ to the region $L$. Symmetrically, the left-velocity $\dot{q}^{-}$, if it exists, belongs to the cone $-\mathrm{V}(\mathrm{q})$. If the proper velocity $\dot{\mathrm{q}}=\dot{\mathrm{q}}^{+}=\dot{\mathrm{q}}^{-}$exists at the considered instant, it belongs to the linear subspace $V(q) \cap-V(q)$ of $E(q)$

We are to study the dynamics of the system, submitted from another part to some given forces, under the hypothesis made explicit in Sect. 2 that the unilateral constraints are frictionless.

Some practical instances have long been discussed; this usually exhibits finite successions of time intervals : when $t$ ranges over the interior of each of these intervals, $\mathrm{J}(\mathrm{q}(\mathrm{t}))$ remains a constant subset of $\{1,2, \ldots, \nu\}$. Let us call this a motion of finite sort. Counter- . examples can be produced where no motion of this sort satisfies the equations of Dynamics ${ }^{1}$. Practising mechanists may consider such instances as pathological and restrict themselves to the search of motions of finite sort ; even so, they will have to face two crucial questions :

Question 1. Starting from an instant $t_{0}$, with $q\left(t_{0}\right)=q_{0} \in L$, $\dot{\mathrm{q}}^{+}\left(\mathrm{t}_{\mathrm{o}}\right)=\dot{\mathrm{q}}_{\mathrm{o}}^{+} \in \mathrm{V}\left(\mathrm{q}_{\mathrm{o}}\right)$, determine which of the contacts $\mathrm{f}_{\alpha}=0, \alpha \in \mathrm{J}\left(\mathrm{q}_{\mathrm{o}}\right)$, persist during a subsequent interval.

Question 2. If some interval during which $\mathrm{f}_{\alpha}(\mathrm{q}(\mathrm{t}))>0$ ends at an ins- 
tant $t_{1}$ such that $f_{\alpha}\left(q\left(t_{1}\right)\right)=0$, a shock is expected to occur; determine the right-velocity $\dot{\mathrm{q}}^{+}\left(\mathrm{t}_{1}\right)$.

E. Delassus showed ${ }^{2}$ that Question 1 had been incorrectly addressed by his eminent predecessors. One usually begins with the tentative assumption that all the contacts $\alpha \in J\left(q_{o}\right)$ such that (1.2) holds as an equality persist ; this amounts to treat the corresponding constraints $\mathrm{f}_{\alpha}=0$ as bilateral and the associated reactions are then calculated from the equations of Dynamics. If the calculation yields for one or more of these reactions a sign incompatible with the unilaterality of the corresponding constraint, the tentative assumption must be rejected and sma1ler subsets of $J\left(q_{0}\right)$ have to be tried in the same way. By very simple counter-examples, Delassus demonstrated that the contacts which cease are not necessarily those corresponding to conflicting signs in the first calculation. Even the existence of some satisfactory subset of $J\left(q_{o}\right)$ is a priori to prove, as well as its uniqueness. Delassus'arguments toward a correct solution seem today difficult to read; a much clearer account of his ideas can be found in ${ }^{3}$ (also annexed to the last edition of ${ }^{4}$ ).

The present author ${ }^{5,6}$ developed a more expedient approach to the same question, using convex optimization. The result may be viewed as the extension of the Gauss-Appe11 "principle of least constraint" to unilateral situations. (The same idea is applied, with an infinite number of degrees of freedom, to the unilaterality of the incompressibility constraint in a liquid in $7,8,9$, papers discussing the inception of cavitation when capillary effect may be neglected).

About Question 2, it is classical that adapting the no-friction hy- 
pothesis to the dynamics of percussions does not yield enough information to determine $\dot{q}^{+}\left(t_{1}\right)$. The shock is classically called elastic if it preserves energy ; adding this assumption is known to determine $\dot{q}^{+}\left(t_{1}\right)$ only in the case of a single contact. ${ }^{10,11}$ However, it is widely recognized today that mechanical models have not necessarily to be deterministic. M. Schatzman 12,13 has effectively studied the dynamics of a system of $\mathrm{fi}^{-}$ nite freedom with frictionless unilateral constraints and elastic shocks, under the convenience assumption that $L$ consists of a convex subset of $\mathbb{R}^{\mathrm{n}}$; her approach is based on regularization techniques. For the case $\nu=1$, see also C. Buttazzo and D. Percivale, ${ }^{14,15}$ who apply the concept of $\Gamma$-convergence to the approximation of solutions. (On the other hand, as an example of problem with infinite freedom, numerous papers have in recent years been devoted to the vibrating string in the presence of an obstacle, initially considered by L. Amerio and G. Prouse ${ }^{16}$; see e.g. ${ }^{17,18}$ Another example is provided by the longitudinal dynamics of a rectilinear bar, an end of which hits an obstacle; see C. Do ${ }^{19}$ ).

In contrast, the present paper rests on the recently introduced concept of a standard inelastic shock, ${ }^{20}$ essentially dissipative, whose properties are discussed in Sect. 5 below. This results (Sect. 8) in a synthetic formulation of the evolution problem, which embodies in particular the jump conditions, in the event of a shock of the said sort, and the equations of dynamics for possible phases of smooth motion (considered in Sect. 3). The formulation may also be turned into a form in which the sweeping process $^{21}$, plays the central role. Sect. 7 summarizes some properties of this process which reveals itself as the basic example of evo- 
lution under unilateral and irreversible conditions, with possible jumps. In view of the jumps, the solutions of the process are defined as vector functions with locally bounded variation ; their time change is thus expressed in terms of vector measures, for which fundamental inequalities are given. The importance of right-continuity in this connection is explained.

However, the availability of an elaborate theory for the sweeping process does not readily solve all questions raised by the present dynamical problem : the existence of solutions, possible uniqueness, approximation procedures are still under investigation.

Assuming standard inelastic shock in calculating the motion of some elementary systems yields conclusions in agreement with common observation (a very simple example is given in Sect. 5) ; physical situations undoubtedly exist, where this concept gives a reasonably accurate description of reality. But, to the author's opinion, the main interest of the concept lies in the internal mathematical consistency of the resulting evolution problem. People facing technological applications may question such an attitude; let us suggest the following answer.

In many domains of applied science, one is dealing with physical laws which, although nonlinear, are smooth enough to admit some linear approximation, after what various corrections, arising from experimental data, may be effected in order to reach a better agreement with reality. In contrast, when facing such situations as unilateral mechanics (or also dry friction or plasticity) one has to treat highly nonlinear, in fact non differentiable, relations. No linear approximation may be used as a first 
approach ; fortunately the tools of convex analysis are able to provide, as in the present paper, a mathematical framework as consistent and a1most as simple as linear analysis ; related to it, a numerical machinery has been previously devised on the purpose of optimization techniques. The solidity of the theoretical core so constructed minimizes the risk of numerical and logical unconsistency when empirical corrections are afterwards added.

In support to the assertion that the quest for mathematical harmony is more than academic decorum, let us recal1 how successful such an attitude has been in theoretical physics, during the past century. 


\section{UNILATERAL REACTIONS}

During a time interval of smooth motion, all actions experienced by the system are expressed in terms of forces. In the framework of analytical dynamics that we adopt in this paper, if the configuration of the system is q, forces are represented as elements of $E^{\prime}(q)$, the cotangent space at the point $q$ to the configuration manifold 2 . For every possible velocity $v \in E(q)$ of the system through the said configuration, the power of a force $f \in E^{\prime}(q)$ is, by definition, the "scalar product" $\langle\mathrm{v}, \mathrm{f}\rangle$, the bilinear form which places the linear spaces $\mathrm{E}(\mathrm{q})$ and $E^{\prime}(q)$ in duality.

In particular, the mechanical realization of the condition $f_{\alpha}(q) \leqslant 0$ involves some force of constraint, or "reaction", $R_{\alpha} \in E^{\prime}(q)$ about which we shall make the following usual assumptions :

$1^{\circ}$ The reaction $R_{\alpha}$ vanishes unless $f_{\alpha}(q)=0$, i.e.

$\alpha \notin \mathrm{J}(\mathrm{q}) \Rightarrow \mathrm{R}_{\alpha}=0$

$2^{\circ}$ The possible "contact" $\mathrm{f}_{\alpha}(\mathrm{q})=0$ is frictionless, i.e. the power $\left\langle\mathrm{v}, \mathrm{R}_{\alpha}\right\rangle$ is zero for every $\mathrm{v} \in \mathrm{E}(\mathrm{q})$ such that $\left\langle\mathrm{v}, \nabla \mathrm{f}_{\alpha}(\mathrm{q})\right\rangle=0$, where $\nabla f_{\alpha}(q) \in E^{\prime}(q)$ denotes the gradient of $f_{\alpha}$ at the point $q$ (non-zero, 
by hypothesis). This is known to be equivalent to

$$
\exists \lambda_{\alpha} \in \mathbb{R} \text { such that } \mathrm{R}_{\alpha}=-\lambda_{\alpha} \nabla \mathrm{f}_{\alpha}(\mathrm{q}) \text {. }
$$

$3^{\circ}$ The direction of $R_{\alpha}$ is such that the above power is $\geqslant 0$ for every $\mathrm{v}$ directed toward the permitted region $\mathrm{f}_{\alpha} \leqslant 0$, i.e.

$$
\lambda_{\alpha} \geqslant 0
$$

The latter involves in particular that no adhesion occurs at any proper contact ; for the case of the unilateral constraint realized by means of some inextensible string, it involves that the string exhibits no stiffness.

Conversely, we shall suppose that every value of $R_{\alpha} \in E^{\prime}(q)$ satisfying conditions (2.1), (2.2), (2.3) is feasible; this means that the physical realization of the considered unilateral constraint suffers no strength limitation.

Therefore, a value $R \in E^{\prime}(q)$ is feasible as the sum of the reactions of the $\nu$ unilateral constraints if and only if

$$
-R \in N(q)
$$

where $N(q)$ denotes the convex cone generated in $E^{\prime}(q)$ by the elements $\nabla f_{\alpha}(q), \alpha \in J(q)$ (by convention reduced to the zero of $E^{\prime}(q)$ if $\mathrm{J}(\mathrm{q})=\phi)$. 
In view of elementary Convex Analysis, $\mathrm{N}(\mathrm{q})$ is a closed convex polyhedral cone, equal to the polar cone, relative to the scalar product $<$,. .> , of the closed convex polyhedral cone $V(q)$ defined in (1.4), i.e.

$$
N(q)=\left\{r \in E^{\prime}(q): \forall v \in V(q),<v, r>\leqslant 0\right\}
$$

and symmetrically, with $\mathrm{N}$ and $\mathrm{V}$ exchanged.

Classically, $\mathrm{N}(\mathrm{q})$ is called the outward normal cone to the region L of 2 at the point $q$. REMARK. Let us discuss more precisely than in Sect. 1 the meaning of $\mathrm{V}\left(\mathrm{q}_{1}\right)$, the so-called tangent cone at some point $\mathrm{q}_{1}$ of $\mathrm{L}$. For each $\alpha \in \mathrm{J}\left(\mathrm{q}_{1}\right)$, condition $<\mathrm{v}, \nabla \mathrm{f}_{\alpha}\left(\mathrm{q}_{1}\right)>\leqslant 0$ is indeed necessary and sufficient for the existence of a motion $t \rightarrow q(t)$ starting from $q_{1}$ with $v$ as initial right-velocity and verifying $f_{\alpha}(q(t)) \leqslant 0$ in the sequel. But the following counter-example show that, some element $v$ being chosen in $\mathrm{V}\left(\mathrm{q}_{1}\right)$, it may prove impossible to construct a motion satisfying all conditions $\mathrm{f}_{\alpha}(\mathrm{q}(\mathrm{t})) \leqslant 0$ together.

Take $\mathrm{n}=3$, with three inequalities

$$
\begin{aligned}
& \mathrm{f}_{1}(\mathrm{q}) \equiv-\mathrm{q}^{1} \leqslant 0 \\
& \mathrm{f}_{2}(\mathrm{q}) \equiv \mathrm{q}^{1}-\mathrm{q}^{2} \mathrm{q}^{3} \leqslant 0 \\
& \mathrm{f}_{3}(\mathrm{q}) \equiv-\mathrm{q}^{2}-\mathrm{q}^{3} \leqslant 0
\end{aligned}
$$

For $\mathrm{q}_{1}=(0,0,0)$, one has $\mathrm{J}\left(\mathrm{q}_{1}\right)=\{1,2,3\}$ and 


$$
\mathrm{v}\left(\mathrm{q}_{1}\right)=\left\{\mathrm{v}=\left(\mathrm{v}^{1}, \mathrm{v}^{2}, \mathrm{v}^{3}\right): \mathrm{v}^{1}=0, \mathrm{v}^{2}+\mathrm{v}^{3} \geqslant 0\right\}
$$

Every motion $t \rightarrow q(t)$ starting from $q_{1}$ at time 0 , with rightvelocity $\mathrm{v}=(0,2,-1) \in \mathrm{V}\left(\mathrm{q}_{1}\right)$ yields for $\mathrm{t}>0$

$$
q^{2}(t)=2 t+o(t), \quad q^{3}(t)=-t+o(t) .
$$

Hence $q^{2}(t) q^{3}(t)=-2 t^{2}+o\left(t^{2}\right)$ is negative in some right-neighborhood of zero; this contradicts inequalities $(2.6),(2.7)$.

The following additional regularity assumption is known to secure equivalence between $\mathrm{v} \in \mathrm{V}(\mathrm{q})$ and the existence of a motion in $\mathrm{L}$, starting from $\mathrm{q}$ with $\mathrm{v}$ as initial right-velocity (cf. ${ }^{22}$; in Optimization Theory, this is called a "qualification" condition) :

$$
\text { interior } \mathrm{V}(\mathrm{q}) \neq \phi
$$

By classical Convex Analysis, this in turn is found equivalent to the existence of a compact base for the polar cone $\mathrm{N}(\mathrm{q})$ of $\mathrm{V}(\mathrm{q})$, i.e. there exists in $E^{\prime}(q)$ a hyperplane, not containing the origin, which intersects all the half-lines generated by the $\nabla f_{\alpha}(q), \alpha \in J(q)$ (this is understood to hold, trivially, if $\mathrm{J}(\mathrm{q})=\phi)$.

The above counter-example leads to question the generality of the so-called Principle of Fourier. This asserts that an element $R$ of $E^{\prime}(q)$ is a feasible value for the total reaction if and only if $<\delta q, R\rangle \geqslant 0$ for every "virtual displacement" (this is another word for 
the velocity of an imagined motion) starting from $q$ and directed into the permitted region L . If condition (2.10) is satisfied, this property is indeed equivalent to $(2.4)$. Examining the region $\mathrm{L}$ of $\mathbb{R}^{3} \mathrm{de}-$ fined by $(2.6),(2.7),(2.8)$, which does not verify condition (2.10) at the point $\mathrm{q}_{1}=(0,0,0)$, throws some light on the situation. The subset $\mathrm{w}$ of $\mathrm{V}\left(\mathrm{q}_{1}\right)$ defined by $\mathrm{v}^{1}=0, \mathrm{v}^{2} \geqslant 0, \mathrm{v}^{3} \geqslant 0$ might be more 1egitimately interpreted as the tangent cone to $\mathrm{L}$ at $\mathrm{q}_{1}$. Whether every element of its polar cone $\mathrm{W}^{\circ}$, larger than $\mathrm{N}\left(\mathrm{q}_{1}\right)$, is a feasible value of the total reaction of the supposedly frictionless unilateral constraints appears as a mechanically irrelevant question. The tangent planes at $\mathrm{q}_{\mathrm{l}}$ to the smooth boundaries defined by equations $\mathrm{f}_{1}=0, \mathrm{f}_{2}=0$, $\mathrm{f}_{3}=0$ make zero angles. This allows for $\mathrm{q}$, considered as a material point, to be "pinched" between these boundaries in the position $\mathrm{q}_{1}$; so infinitely large values of the normal components of the boundary reactions may arise as a response to some moderate driving force acting on $q$. Under such circumstances, however small may be the friction coefficient between $\mathrm{q}$ and the boundaries, friction cannot be neglected.

Points at which $(2.10)$ is not satisfied are not necessarily isolated : for instance, in $\mathbb{R}^{3}$, some boundaries may meet at zero angle all along a curve. The discussion of would-be frictionless bilateral constraints in classical Analytical Mechanics exhibits similar "pathological" situations ; see e.g. ${ }^{11}$, Sect. 9.2.b. 


\section{DYNAMICAL EQUATIONS OF SHOCKLESS MOTION}

In addition to the reactions of constraints, the system is supposed to experience some configuration-dependent forces, represented, in our setting of analytical dynamics, by giving the coefficients $Q_{i}$ of the differential form "virtual work" of this system of forces, as continuous functions of $\left(q^{1}, \ldots, q^{n}\right)$; this amounts to define, on the manifold 2 , a continuous field of covectors, say $q \rightarrow Q(q) \in E^{\prime}(q)$, possibly dependent also on time.

On the other hand, the expression $\dot{T}(q, \dot{q})$ of the kinetic energy is given ; for simplicity's sake we restrict ourselves in this paper to the scleronomic case, implying that $T$ does not contain $t$ as independent variable and is a quadratic form relatively to $\dot{q} \in E(q)$.

Then the system of Lagrange equations for every smooth motion writes down as

$$
P=Q+R
$$

where $P$ denotes, as classical, the element of $E^{\prime}(q)$ whose components, relative to the chosen parametrization of $Q$ are 


$$
P_{i}=\frac{d}{d t} \frac{\partial T}{\partial \dot{q}^{i}}-\frac{\partial T}{\partial q} .
$$

Eliminating $R$ through (2.4), we give (3.1) the form of a second order differential inclusion

$$
Q(t, q(t))-P(q(t), \dot{q}(t), \ddot{q}(t)) \in N(q(t)) \text {. }
$$

By a solution of (3.3) over some, possibly unbounded, time interval $I$, we mean a differentiable motion $t \rightarrow q(t)$ such that the $n$ derivatives $t \rightarrow \dot{q}^{i}(t)$ are absolutely continuous functions on every compact subinterval of $I$, with derivatives $t \rightarrow \ddot{q}^{i}(t)$ satisfying (3.3) up to the possible exception of a Lebesgue-negligible subset of I . If I possesses an origin $t_{0}$ and contains it, this implies the existence of the right-derivatives $\dot{q}^{i+}\left(t_{0}\right)$ and makes the initial conditions $q\left(t_{0}\right)=q_{0}$ given in $\mathrm{L}, \dot{\mathrm{q}}^{+}\left(\mathrm{t}_{\mathrm{o}}\right)=\dot{\mathrm{q}}_{\mathrm{o}}$ given in $\mathrm{V}\left(\mathrm{q}_{\mathrm{o}}\right)$, meaningful.

If we put the natural convention

$$
N(q)=\phi \quad \text { for } \quad q \notin L,
$$

the requirement $\mathrm{q}(\mathrm{t}) \in \mathrm{L}$ for every $\mathrm{t}$ in $\mathrm{I}$ is involved in (3.3).

We shall prove now that every solution of (3.3) in the above sense, actually satisfies a somewhat stronger differential inclusion.

The assumptions made imply that the velocity $\dot{q} \in E(q)$ exists for every $t$ in the interior of $I$; since the motion $t \rightarrow q(t)$ takes place in the region $L$, it has been observed in Sect. 1 that $\dot{q} \in V(q) \cap-V(q)$. 
In view of (2.5), we conclude that, for every $t$ in the interior of $I$ and such that (3.3) holds, one has

$$
<\dot{\mathrm{q}}, \mathrm{Q}-\mathrm{P}>=0
$$

For every subset A of a linear space, we shall denote by $\psi_{\mathrm{A}}$ or $\psi(\mathrm{A},$.$) the indicator function of \mathrm{A}$, i.e.

$$
\psi(\mathrm{A}, \mathrm{x})=0 \text { if } \mathrm{x} \in \mathrm{A}, \quad+\infty \text { if } \mathrm{x} \notin \mathrm{A}
$$

This function is convex (resp. lower semi-continuous) if and only if the set $A$ is convex (resp. closed). For a pair of mutually polar convex cones, such as $V(q)$ and $N(q)$ above, the respective indicators $\psi(V,$. and $\psi(\mathrm{N},$.$) are known to constitute a pair of Fenchel conjugate functions.$ Now, (3.3) means that $\psi(\mathrm{N}(\mathrm{q}), \mathrm{Q}-\mathrm{P})=0$. Then, in view of (3.5) and of the fact that $\dot{q} \in \mathrm{V}(\mathrm{q})$, one has, for almost every $t$ in $I$, the equality

$$
\psi(\mathrm{V}(\mathrm{q}), \dot{\mathrm{q}})+\psi(\mathrm{N}(\mathrm{q}), \mathrm{Q}-\mathrm{P})-<\dot{\mathrm{q}}, \mathrm{Q}-\mathrm{P}>=0
$$

expressing that $\dot{q}$ in $E(q)$ and $Q-P$ in $E^{\prime}(q)$ are conjugate points relative to the above pair of conjugate functions. This in turn is known to be equivalent to

$$
Q(t, q)-P(q, \dot{q}, \ddot{q}) \in \partial \psi(V(q), \dot{q}),
$$


where the subdifferential $\partial \psi(\mathrm{V}(\mathrm{q}), \dot{\mathrm{q}})$ classically equals the outward normal cone at the point $\dot{q}$ to the closed convex set $V(q)$. This normal cone is essentially a subset of $\mathrm{N}(\mathrm{q})$; here again, convention (3.4) makes that $\mathrm{q}(\mathrm{t}) \in \mathrm{L}$ is involved in (3.6).

REMARK. The dynamics of supposedly shockless motions in the presence of scleronomic frictionless unilateral constraints, as developed here, exhibits the same reversibility in time as the traditional bilaterally constrained case. In fact the above reasoning could also yield symmetrically

$$
Q(t, q)-P(q, \dot{q}, \ddot{q}) \in-\partial \psi(-V(q), \dot{q})
$$

On the other hand, (3.5) expresses that the total reaction $R \in E^{\prime}(q)$ develops a zero power in the actual motion. The assumptions made imply that the function $t \rightarrow T(q(t), \dot{q}(t))$ is absolutely continuous on every compact subinterval of $I$, hence differentiable almost everywhere. A classical calculation, based on the fact that $T(q, \dot{q})$ is a quadratic form in its second argument, yields the "energy equation"

$$
\frac{d}{d t} T(q(t), \dot{q}(t))=\langle\dot{q}(t), Q(t, q(t))\rangle \text {. }
$$

It permits to establish a priori bounds of $\dot{q}$ for supposed solutions of the initial value problem. This equation is specially useful when the field of covectors $q \rightarrow Q(t, q)$ derives from a time-independent potential function $q \rightarrow W(q)$, i.e. $Q(q)=-\nabla W(q) ;$ then it comes that $\mathrm{T}(\mathrm{q}, \dot{\mathrm{q}})+W(\mathrm{q})$ is a constant of the motion ; this is the familiar conservation property of the total energy. 


\section{SHOCK DYNAMICS}

If an interval of smooth motion ends at some instant $t_{s}$ such that the left-velocity $\dot{q}^{-}\left(t_{s}\right)$, more shortly denoted by $\dot{q}_{s}^{-}$, does not belong to $\mathrm{V}\left(\mathrm{q}_{\mathrm{s}}\right), \mathrm{q}_{\mathrm{s}}=\mathrm{q}\left(\mathrm{t}_{\mathrm{s}}\right)$, a shock necessarily occurs. Classically, by integrating the Lagrange equation (3.1) over the "infinitely short" duration of this shock, one obtains

$$
\mathrm{p}_{\mathrm{s}}^{+}-\mathrm{p}_{\mathrm{s}}^{-}=\Pi
$$

Here $\mathrm{p}_{\mathrm{s}}^{+}$and $\mathrm{p}_{\mathrm{s}}^{-}$respectively denote the right and left limits at time $t_{s}$ of the momentum

$$
p=\frac{\partial T}{\partial \dot{q}} \in E^{\prime}(q)
$$

The percussion of constraint $\Pi \in E^{\prime}\left(q_{s}\right)$ is introduced as the integral, over the shock duration, of the "infinitely large" reaction $R(t)$; as this reaction is supposed to satisfy (2.4) with $N(q)=N\left(q_{\dot{\mathbf{s}}}\right)$, a closed convex cone which does not vary during the shock, one has

$$
-\Pi \in N\left(q_{s}\right)
$$


(a discussion of this argument may be found in ${ }^{11}$, Sect. 9.7.c, Remarque). On the other hand, the kinematical condition

$$
\dot{\mathrm{q}}_{\mathrm{s}}^{+} \in \mathrm{v}\left(\mathrm{q}_{\mathrm{s}}\right)
$$

holds as before.

Even in the special case of a single contact, i.e. $J\left(q_{s}\right)$ consisting of a singleton, conditions (4.1), (4.3) and (4.4) are we 11 known 10,11 to bring insufficient information to derive $\dot{q}_{S}^{+}$from the data $q_{s}$ and $\dot{q}_{s}^{-}$. The parametrization $\left(\mathrm{q}^{\mathrm{i}}\right)$ of the configuration manifold is supposed kinetically regular, in the sense that the quadratic form defined on each tangent space $\mathrm{E}(\mathrm{q})$ by

$$
v \rightarrow 2 T(q, v)=\sum_{i, j} a_{i j}(q) v^{i} v^{j}
$$

(with $a_{i j}=a_{j i}$ ) is positive definite. Let us eccuip the linear space E(q) with a Euclidean structure by taking $2 \mathrm{~T}(\mathrm{q}, \mathrm{v})$ as the definition of the squared norm $\|v\|^{2}$. Equivalently, the Euclidean scalar product of two elements $v$ and $w$ of $E(q)$ is expressed by

$$
v \cdot w=\sum_{i, j} a_{i j}(q) v^{i} w^{j}
$$

For geometrical and notational simplicity, we shall perform the classical trick of using this Euclidean structure of the linear space $E(q)$ in order to identify it with its dual space $E^{\prime}(q)$. From the standpoint of calculation, this means the following : to each choice of a parametri- 
zation $\left(\mathrm{q}^{\mathrm{i}}\right)$ of the manifold $\Omega$ corresponds a base in the tangent space $E(q)$, say $\left(e_{i}\right), i=1, \ldots, n$. The derivatives $\dot{q}^{i}$ of the functions $t \rightarrow q^{i}(t)$ representing some motion constitute, as before, the components relative to this base of the velocity vector $\dot{q}$. On the other hand, in view of (4.5), the expressions

$$
p_{i}=\frac{\partial T}{\partial \dot{q}_{i}}=\sum_{j} a_{i j}(q) \dot{q}^{j}
$$

equal the covariant components of the same element $\dot{q}$ of $E(q)$ relative to the said base, i.e. the respective scalar products $\dot{q} \cdot e_{i}$. The identification trick amounts to declaring that the element $\dot{q}$ of $E(q)$ and the element $p$ of $E^{\prime}(q)$ constitute the same object.

Similarly, the partial derivatives $\partial f_{\alpha} / \partial q^{i}$ are interpreted as the covariant components of the gradient $\nabla f_{\alpha}(q)$, now considered as an element of the Euclidean linear space $E(q)$. We continue to denote by $N(q)$ the convex cone generated by the $\nabla f_{\alpha}(q), \alpha \in J(q)$; this is now a closed convex polyhedral cone in $\mathrm{E}(\mathrm{q})$, actually the polar cone of $\mathrm{V}(\mathrm{q})$ since (2.5) still holds with $\langle\mathrm{v}, \mathrm{r}\rangle$ equal to the Euclidean scalar product $\mathrm{v} \cdot \mathrm{r}$.

In view of the above identification, (4.1) takes on the form

$$
\dot{q}_{s}^{+}-\dot{q}_{s}^{-}=\Pi
$$

while (4.3) and (4.4) stay unchanged.

The shock is traditionally called elastic if it preserves energy, 
that is, in terms of the Euclidean norm of $E\left(q_{s}\right)$,

$$
\frac{1}{2}\left\|\dot{\mathrm{q}}_{\mathrm{s}}^{+}\right\|^{2}=\frac{1}{2}\left\|\dot{\mathrm{q}}_{\mathrm{s}}^{-}\right\|^{2}
$$

In the special case where $J(q)$ reduces to a singleton, say $J(q)=\{1\}$, conditions (4.3), (4.4), (4.6) and (4.7) are found, by elementary geometry, equivalent to : the vector $\dot{q}_{s}^{+}$equals the mirror image of the vector $\dot{q}_{s}^{-}$relative to the hyperplane of $E\left(q_{s}\right)$ with normal $\nabla f_{1}\left(q_{s}\right)$.

On the other hand, for the same special case $J(q)=\{1\}$, the shock is called soft or inelastic if, instead of (4.7), one has

$$
\dot{q}_{s}^{+} \cdot \nabla f_{1}\left(q_{s}\right)=0
$$

We propose, in the Section to come, a generalization of the latter. 


\section{STANDARD INELASTIC SHOCK}

Let us first recall a few facts of elementary convex analysis in a Euclidean linear space E (also valid in an infinite-dimensional real Hilbert space). For every nonempty closed convex subset C of $\mathrm{E}$, every point $z$ of $E$ possesses a unique proximal point in $C$, denoted here by $\operatorname{prox}(\mathrm{C}, \mathrm{z})$. Then (cf. ${ }^{23}$ or, for more generality, ${ }^{24}$ ) the following non-linear generalization of the classical decomposition of $E$ into the sum of orthogonal subspaces holds :

LEMMA OF THE TWO CONES. If $\mathrm{V}, \mathrm{N}$ denote a pair of mutually polar closed convex cones in $E$ and if $x, y, z$ are three points of $E$, assertions i) and ii) below are equivalent :

i) $\quad x=\operatorname{prox}(V, z), y=\operatorname{prox}(N, z)$

ii) $z=x+y, x \in V, y \in N, x \cdot y=0$.

COROLLARY

$\mathrm{x}=\operatorname{prox}(\mathrm{V}, \mathrm{z}) \Longleftrightarrow \mathrm{z}-\mathrm{x}=\operatorname{prox}(\mathrm{N}, \mathrm{z})$

Using again the setting of Sect. 4, let us propose :

DEFINITION. The shock at time $t_{s}$ is said standard inelastic if the three following conditions, equivalent in view of $(4.3),(4.4),(4.6)$, hold 


$$
\begin{aligned}
\dot{\mathrm{q}}_{\mathrm{s}}^{+} & =\operatorname{prox}\left(\mathrm{V}\left(\mathrm{q}_{\mathrm{s}}\right), \dot{\mathrm{q}}_{\mathrm{s}}^{-}\right) \\
-\Pi & =\operatorname{prox}\left(\mathrm{N}\left(\mathrm{q}_{\mathrm{s}}\right), \dot{\mathrm{q}}_{\mathrm{s}}^{-}\right) \\
\Pi \cdot \dot{\mathrm{q}}_{\mathrm{s}}^{+} & =0 .
\end{aligned}
$$

Equivalence immediately results from the above Lemma, by taking $z=\dot{q}_{s}^{-}, \quad x=\dot{q}_{s}^{+}, \quad y=-\Pi$.

Condition (5.1) presents the reassuring aspect of an economy principle : among all the values of $\dot{q}_{s}^{+}$kinematically compatible with the unilateral constraints, it imposes the nearest one to $\dot{q}_{S}^{-}$, in the sense of the kinetic metric of $\mathrm{E}\left(\mathrm{q}_{\mathrm{s}}\right)$.

Symmetrically, (5.2) may be written as

$$
\Pi=\operatorname{prox}\left(-\mathrm{N}\left(\mathrm{q}_{\mathrm{S}}\right),-\dot{\mathrm{q}}_{\mathrm{S}}^{-}\right)
$$

Using the equations of the dynamics of percussions under the form (4.6), one sees that $-\dot{q}_{s}^{-}$equals the percussion which should be applied to the system in order to obtain $\dot{\mathrm{q}}_{\mathrm{S}}^{+}=0$. Then (5.4) expresses that, in the set of the values of $\Pi$ permitted by the law (4.3) of frictionless unilaterality, the actual solution consists in the nearest point to this stopping percussion.

Concerning condition (5.3), one gives it in view of (4.6) the equivalent form

$$
\frac{1}{2}\left\|\dot{q}_{s}^{+}\right\|^{2}-\frac{1}{2}\left\|\dot{q}_{s}^{-}\right\|^{2}=-\frac{1}{2}\left\|\dot{q}_{s}^{-}-\dot{q}_{s}^{+}\right\|^{2}
$$


This displays a loss of kinetic energy : the process described by the above definition is essentially dissipative. Observe that (5.3) holds in particular if $\dot{\mathrm{q}}_{\mathrm{S}}^{+}$happens to be kinematically consistent with the permanence of all the contacts $\mathrm{f}_{\alpha}=0, \alpha \in \mathrm{J}\left(\mathrm{q}_{\mathrm{s}}\right)$. Hence (5.5) may be viewed as a generalization of a classical theorem of Carnot, pertaining to the sudden introduction of persistent, bilateral, constraints. ${ }^{11}$

In that connection, when the cone $\mathrm{V}\left(\mathrm{q}_{\mathrm{S}}\right)$ is given, the mapping $\dot{\mathrm{q}}_{\mathrm{S}}^{-} \rightarrow-\Pi$ defined by (5.2) appears as a relation between some "velocity" and some "force" of the form currently called a standard dissipative process 25,26 ; in fact this mapping equals the gradient of some convex function, namely $v \rightarrow\left(\operatorname{dist}\left(v, v_{s}\right)\right)^{2} / 2$.

EXAMPLE. Let the system consist of a single particle moving in some vertical plane, with $\left(\mathrm{q}^{1}, \mathrm{q}^{2}\right)$ as orthonormal Cartesian coordinates, the $\mathrm{q}^{2}$ axis vertical and oriented upward. Fixed frictionless boundaries are assumed to impose

$$
\mathrm{q}^{2} \geqslant 0 \quad, \quad \mathrm{q}^{1} \cos \theta+\mathrm{q}^{2} \sin \theta \leqslant 0
$$

with $\theta$ given in $]-\frac{\pi}{2},+\frac{\pi}{2}[$.

A phase of motion :

$$
t<0, \quad q^{1}(t)=w t, \quad q^{2}(t)=0
$$

( $w>0$ constant) ends with a shock at time $t_{s}=0, q_{s}=(0,0)$, since the left-velocity $\dot{\mathrm{q}}_{\mathrm{S}}^{-}=(\mathrm{w}, 0)$ does not belong to 


$$
\mathrm{v}\left(\mathrm{q}_{\mathrm{s}}\right)=\left\{\mathrm{v} \in \mathbb{R}^{2}: \mathrm{v}^{2} \geqslant 0, \mathrm{v}^{1} \cos \theta+\mathrm{v}^{2} \sin \theta \leqslant 0\right\} .
$$

Here the kinetic norm coincides with the natural Euclidean norm of the plane. Using (5.1) to determine $\dot{\mathrm{q}}_{\mathrm{s}}^{+}$leads to distinguish between two cases.
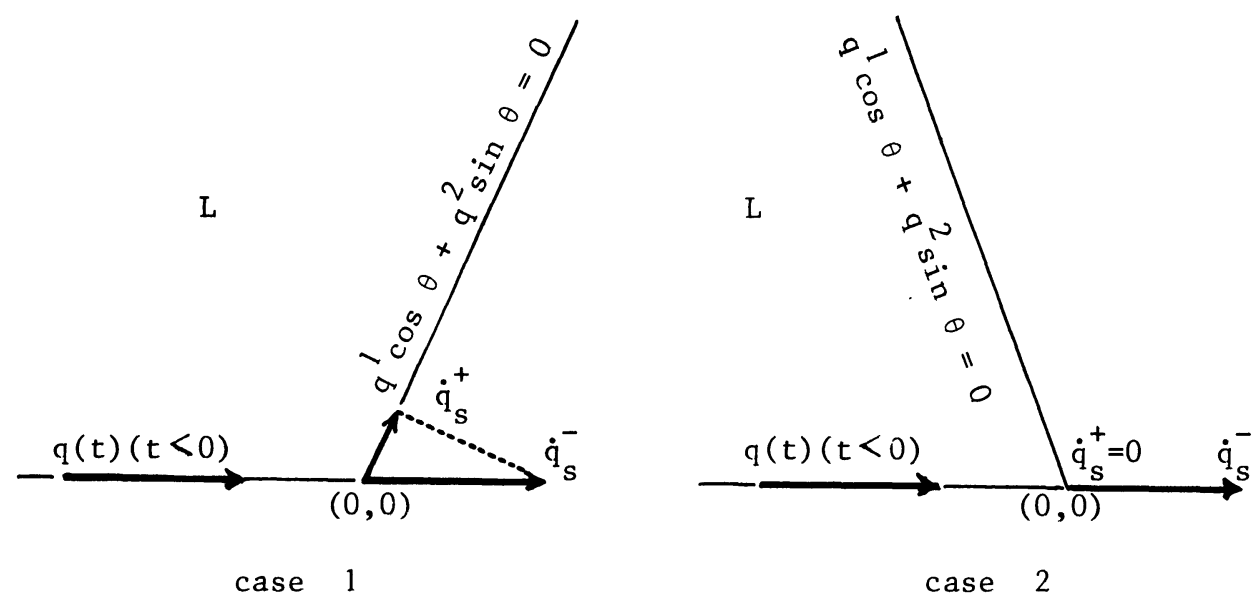

Case 1 : $-\frac{\pi}{2}<\theta<0$. The subsequent motion takes place on the ascendent boundary line, with initial velocity :

$$
\dot{\mathrm{q}}_{\mathrm{s}}^{+}=\left(\mathrm{w} \sin ^{2} \theta,-\mathrm{w} \sin \theta \cos \theta\right)
$$

Case 2 $: 0 \leqslant \theta<\frac{\pi}{2}$. The subsequent motion is rest.

REMARK. Minimizing over $\mathrm{V}\left(\mathrm{q}_{s}\right)$ the distance to $\dot{\mathrm{q}}_{S}^{-}$is the same as minimizing the square of this distance; hence the determination of $\dot{q}_{S}^{+}$from (5.1) constitutes a problem of "quadratic programming". Let us only observe that the base $\left(e_{i}\right)$ in $E\left(q_{s}\right)$ corresponding to some parametrization $\left(q^{i}\right)$ of 2 has no reason to be orthonormal relatively to the Euclidean 
structure precedingly defined; in fact (4.5) shall be used to express the squared norm. Symmetrically, (5.2) makes the determination of $I$ a problem of quadratic programming; however it should be kept in mind that the elements $\nabla f_{\alpha}\left(q_{s}\right), \alpha \in J\left(q_{s}\right)$, generating the convex polyhedral cone $N\left(q_{s}\right)$ possess the respective partial derivatives $\frac{\partial f}{\partial q^{i}}\left(q_{s}\right)$ as covariant components relative to the above base, i.e. such a derivative equals the scalar product of $\nabla f_{\alpha}\left(q_{s}\right)$ by the base vector $e_{i}$. If calculations are to be performed by using this sort of components, recall that, for a pair of vectors $v$, w with covariant components $v_{i}, w_{j}$, the scalar product is expressed by

$$
v \cdot w=\sum_{i, j} a^{i j} v_{i} w_{j}
$$

where $a^{i j}$ is the inverse matrix of $a_{i j}$. 


\section{DIFFERENTIAL MEASURES}

Let I denote a real interval ; for brevity, we shall restrict the sequel to the case where $I$ is open on the right, possibly unbounded, but is closed on the left, with origin $t_{0}$. A function $f$ of $I$ into a real Banach space $X$ is said to have a locally bounded variation if it has a bounded variation, in the sense of the norm of $\mathrm{X}$, over every compact subinterval of $\mathrm{I}$; notation $\mathrm{f} \in \operatorname{lbv}(\mathrm{I}, \mathrm{X})$.

This classically implies the existence of a measure $\mathrm{df}$ on $\mathrm{I}$, with values in $X$, called the differential measure of $f$, with the characteristic property that, for every compact subinterval [a,b] of I,

$$
\int_{[a, b]} d f=f^{+}(b)-f^{-}(a) .
$$

The existence of the right and left limits $f^{+}$and $f^{-}$is secured by the bounded variation assumption ; by convention, $\mathrm{f}^{-}\left(t_{0}\right)$ is taken equal to $f\left(t_{0}\right)$. In particular, by making $a=b,(6.1)$ yields that every discontinuity point of $f$ constitutes an atom of the vector measure df, with a mass equal to the jump of $f$.

Incidentally for every open subset $\Omega$ of $I$, the restriction of the measure df to $\Omega$ constitutes the derivative, in the sense of 
Schwartz's distributions in $\Omega$, of the vector measure $\mathrm{f} d t$ (dt : the Lebesgue measure in $\Omega$ ).

From (6.1) it ensues that, for every $t \in I$,

$$
\begin{aligned}
& f^{+}(t)=f\left(t_{0}\right)+\int_{\left[t_{0}, t\right]} d f \\
& f^{-}(t)=f\left(t_{0}\right)+\int_{\left[t_{0}, t[\right.} d f .
\end{aligned}
$$

Hence, knowing the measure df only allows one to reconstruct $\mathrm{f}^{+}$and $\mathrm{f}^{-}$but not the actual function $\mathrm{f}$; this shows that the familiar pattern, according to which the "differential" df determines $f$ up to an additive constant can subsist in the present context only under some assumption connecting $f$ with its one-side limits. For a vast class of evolution problems, formulated in terms of differential measures, the good assumption turns out to be $f=\mathrm{f}^{+}$, i.e. $\mathrm{f}$ right-continuous ; notation $f \in \operatorname{rclbv}(I, X)$. This has the immediate advantage of making, for such an evolution problem, the initial condition $f\left(t_{0}\right)=f_{0}$ meaningful ; the following goes a little deeper in explaining the situation.

Let $B: X \times X \rightarrow \mathbb{R}$ denote a norm-continuous bilinear form. For every couple $f, g$ of elements of $\operatorname{lbv}(I, X)$ the function $t \rightarrow B(f(t), g(t))$ belongs to $\operatorname{lbv}(I, \mathbb{R})$ and the following calculation rules holds to express its differential measure : 27,28

$$
\begin{aligned}
\mathrm{dB}(\mathrm{f}, \mathrm{g}) & =\mathrm{B}\left(\mathrm{df}, \mathrm{g}^{+}\right)+\mathrm{B}\left(\mathrm{f}^{-}, \mathrm{dg}\right) \\
& =\mathrm{B}\left(\mathrm{df}, \mathrm{g}^{-}\right)+\mathrm{B}\left(\mathrm{f}^{+}, \mathrm{dg}\right)
\end{aligned}
$$


Under some general definition that we shall not develop here, the symbols on the right-hand side represent real (signed) measures ; in usual instances, in particular when $\mathrm{X}$ is finite-dimensional or is a Hilbert space, a differential measure, such as df above, possesses a density relative to some nonnegative real measure $d \mu$, i.e. there exists a function $f^{\prime} \subset \mathscr{L}_{1 o c}^{1}(I, d \mu ; X)$ such that $d f=f^{\prime} d \mu$. Then the said definition is found to yield

$$
B\left(d f, g^{+}\right)=B\left(f^{\prime}, g^{+}\right) d \mu
$$

a meaningful expression since $\mathrm{g}^{+}$, as every element of $1 \mathrm{bv}(\mathrm{I}, \mathrm{X})$, is locally bounded and universally measurable.

Let us make now the additional assumptions that the bilinear form $B$ is symmetric and that the quadratic form $x \rightarrow B(x, x)$ it generates is nonnegative. Then it is proved that, for every $f \in 1 b v(I, X)$, the following inequalities hold, in the sense of the ordering of real (signed) measures

$$
2 B\left(f^{-}, d f\right) \leqslant d B(f, f) \leqslant 2 B\left(f^{+}, d f\right)
$$

The inequality on the right explains the importance of the assumption $f=f^{+}$, i.e. $f \in \operatorname{rclbv}(I, X)$, when "energy inequalities" for the considered'evolution problems are to be derived; see Sect. 7 below. 


\section{THE SWEEPING PROCESS}

Here is a basic evolution problem of the unilateral sort, intensive1y studied by the author, with the theory of elastoplastic systems as primary motivation. ${ }^{21,25}$ This problem will be shown in the sequel to present a close connection with the subject of this paper.

Let be given a moving set $t \rightarrow C(t)$, or multifunction from the interval I into some real Hilbert space $H$, with closed convex values. A moving point $\mathrm{u}: \mathrm{I} \rightarrow \mathrm{H}$ is called a solution of the sweeping process by $C$ if it satisfies the differential inclusion

$$
-\dot{u}(t) \in \partial \psi(C(t), u(t))
$$

At the first level of the theory, the time-derivative $\dot{u}(t)$, i.e. the velocity vector of the moving point, is defined in the elementary way and (7.1) is supposed to hold for every $t$ in $I$. If $C(t)$ possesses a non-empty interior, a particularly suggestive interpretation of (7.1) may be given : as long as $u(t)$ lies in this interior, the set $\partial \psi(C(t), u(t))$, namely the outward normal cone to $C(t)$ at this point, reduces to the zero of $\mathrm{H}$; then (7.1) implies that the point $\mathrm{u}$ stays at rest. It is only when caught up with by the boundary of $C(t)$ that $u$ takes up a 
motion, in some inward normal direction so as to go on belonging to the moving set ; in fact the right-hand side of ( 7.1 ) would be empty if $\mathrm{u}(\mathrm{t}) \notin \mathrm{C}(\mathrm{t})$

The existence of differentiable or absolutely continuous solutions to (7.1) clearly requires some smoothness assumptions concerning the motion of $\mathrm{C}$; they can be formulated, for instance, in terms of the Hausdorff distance between successive positions of the moving set in $H$. At a more elaborate level, some "unilateral" rating for the evolution of $\mathrm{C}$ is put forward, sensitive only to retraction. 29

If, on the contrary, the multifunction $t \rightarrow C(t)$ is discontinuous, jumps are expected to occur in the motion of $u$, so that condition (7.1) has to be given some extended meaning :

DEFINITION. We sha11 say that $u: I \rightarrow H$ is a solution of the sweeping process by $C$ in the sense of differential measures if $u \in \operatorname{rclbv}(I, H)$ and if there exists (non uniquely) a nonnegative real measure $\mathrm{d} \mu$, with a vector function $u^{\prime}{ }_{\mu} \in \mathcal{L}_{10 c}^{l}(\mathrm{I}, \mathrm{d} \mu ; \mathrm{H})$, such that $d u=u_{\mu}^{\prime} d \mu$ and that

$$
-u_{\mu}^{\prime}(t) \in \partial \psi(C(t), u(t))
$$

holds for every $t$ in $I$.

As usual, we denote with $\mathcal{L}$ the non-separated topological linear spaces consisting of everywhere defined functions, while $L$ refers to the corresponding separated spaces whose elements are equivalence classes of such functions. 
Observe that (7.2) implies $u(t) \in C(t)$ for every $t \in I$ : otherwise the right-hand side would be empty. Instead of requiring of (7.2) to be satisfied everywhere, one could equivalently except a possible d $\mu-n e g 1 i-$ gible subset $S$ of $I$, provided the condition $u(t) \in C(t)$ is additionally prescribed everywhere in I ; in fact the latter ensures that the right-hand side contains at least the zero of $H$, hence the latitude of correcting $u^{\prime}{ }_{\mu}$ in $S$, so as to finally satisfy (7.2) for every $t$.

A prominent feature is that, if $u$ complies with the above Definition, the same holds when replacing $d \mu$ by any other nonnegative real measure $d v$, relatively to which the vector measure du possesses a "density" $u^{\prime}{ }_{\nu} \in \mathcal{L}_{10 c}^{l}(\mathrm{I}, \mathrm{d} \nu ; \mathrm{H})$. This easily results from the fact that every set $\partial \psi(C(t), u(t))$ is a cone : use the sum $d \mu+d \nu=d \beta$ as a "base" measure, relatively to which $d \mu$ and $d \nu$ both possess nonnegative density functions $\mu_{\beta}^{\prime}$ and $\nu_{\beta}^{\prime}$; observe that every subset of I throughout which $\nu_{\beta}^{\prime}=0$ is dv-negligible.

In particular, with the vector measure du it is associated the non-negative real measure $|d u|$, called its absolute value, ${ }^{30}$ and du possesses a density relatively to it (this is from elementary measure theory if $\mathrm{H}$ is finite-dimensional, but also holds for an arbitrary Hilbert space : such a space is said to possess the Radon-Nikodym property). So $\mathrm{d} \mu$ in the above Definition may equivalently be taken equal to $|\mathrm{du}|$. Let us now give an illustration of the importance of the rightcontinuity involved in the requirement $u \in$ rclbv. Let $u$ and $\bar{u}$ denote two solutions of the sweeping process by $C$, satisfying (7.2) with $\mathrm{du}=\mathrm{u}_{\mu}^{\prime} \mathrm{d} \mu$ and $\overline{\mathrm{u}}=\overline{\mathrm{u}}_{\mu}^{\prime}-\mathrm{d}_{\mu}$ respectively. The nonnegative measures $\mathrm{d} \mu$ 
and $d \bar{\mu}$ possess nonnegative densities relatively to $d \beta=d \mu+d \bar{\mu}$; hence, using again the fact that every $\partial \psi$ is a cone, one obtains

$$
\begin{aligned}
& -u_{\beta}^{\prime}(t) \in \partial \psi(C(t), u(t)) \\
& -\bar{u}_{\beta}^{\prime}(t) \in \partial \psi(C(t), \bar{u}(t)) .
\end{aligned}
$$

Classically, for every $t$, the multifunction $x \rightarrow \partial \psi(C(t), x)$ from $H$ into itself is monotone; therefore

$$
\left(\bar{u}_{\beta}^{\prime}(t)-u_{\beta}^{\prime}(t)\right) \cdot(\bar{u}(t)-u(t)) \leqslant 0 .
$$

The scalar product of $\mathrm{H}$, denoted here by . , may be taken as the bilinear form $B$ in the right-hand inequality (6.6). Due to the rightcontinuity of $u$ and $\bar{u}$, this yields that the real measure $d\|\bar{u}-u\|^{2}$ is nompositive. Consequently, for every pair $u, \bar{u}$ of solutions of the sweeping process by $C$, the distance $\|\bar{u}-u\|$ is a nonincreasing function of $t$; this implies in particular that at most one such solution may agree with some initial condition $u\left(t_{0}\right)=u_{0} \in C\left(t_{0}\right)$.

Concerning the existence of this solution, see ${ }^{21}$.

In the above definition of the sweeping process is involved a precise jump condition for every discontinuity point $t_{s}$ of a solution $u$. In fact, at such a point, the vector measure du presents an atom of value 


$$
u\left(t_{s}\right)-u^{-}\left(t_{s}\right)=u_{\mu}^{\prime}\left(t_{s}\right) \mu_{s}
$$

where $\mu_{s}>0$ denotes the value of the corresponding atom of $d \mu$. Then (7.2) implies

$$
u^{-}\left(t_{s}\right)-u\left(t_{s}\right) \in \partial \psi\left(C\left(t_{s}\right), u\left(t_{s}\right)\right)
$$

Due to the classical characterization of proximal points in a Hilbert space, this is equivalent to

$$
u\left(t_{s}\right)=\operatorname{prox}\left(C\left(t_{s}\right), u^{-}\left(t_{s}\right)\right.
$$

This jump law is to be compared with a natural time-discretization procedure for approximating solutions. Let us start with the elementary formulation (7.1), involving the existence of the time derivative $\dot{u}$. An increasing sequence of points $t_{i}$ is chosen in $I$ and the vector

$$
\frac{1}{t_{i+1}-t_{i}}\left(\hat{u}\left(t_{i+1}\right)-\hat{u}\left(t_{i}\right)\right)
$$

is adopted as an approximant of the derivative at point $t_{i+1}$ of some approximate solution $\hat{\mathrm{u}}$. Then replacing (7.1) by

$$
\frac{1}{t_{i+1}-t_{i}}\left(\hat{u}\left(t_{i}\right)-\hat{u}\left(t_{i+1}\right)\right) \in \partial \psi\left(c\left(t_{i+1}, \hat{u}\left(t_{i+1}\right)\right)\right.
$$

constitutes a discretization scheme of the implicit sort. Because $\partial \psi$ is 
a cone, the positive factor $1 / t_{i+1}-t_{i}$ may be dropped, hence equivalently

$$
\hat{\mathrm{u}}\left(\mathrm{t}_{\mathrm{i}+1}\right)=\operatorname{prox}\left(\mathrm{C}\left(\mathrm{t}_{\mathrm{i}+1}\right), \hat{\mathrm{u}}\left(\mathrm{t}_{\mathrm{i}}\right)\right) \text {, }
$$

a relation similar to the jump condition (7.3). In fact the above approximation procedure amounts to replace the given moving set $t \rightarrow C(t)$ by another one which moves stepwise, say $t \rightarrow \hat{c}(t)$, with the constant value $\hat{C}(t)=C\left(t_{i}\right)$ for every $t \in\left[t_{i}, t_{i+1}[\right.$. The corresponding solution $\hat{u}$ of the sweeping process, agreeing with the initial condition $\left.\hat{u}_{(} t_{0}\right)=u_{0}$ is a right-continuous step-function whose successive values are inductively constructed by means of (7.4). This procedure may be called the catching-up algorithm, since the moving point $\hat{u}(t)$, instead of being swept by $C(t)$, is left at rest during the time-interval $\left[t_{i}, t_{i+1}[\right.$, at the end of which it has to catch up with $C\left(t_{i+1}\right)$ by the shortest way.

In the case of a smoothly moving set $C$, one obtains the uniform convergence of $\hat{u}$ toward the exact solution when the subdivision $\left(t_{i}\right)$ is uniformely refined. ${ }^{25}$ In the discontinuous case, the uniform approximation of $u$ by $\hat{u}$ can visibly be expected only if one includes in the sequence $\left(t_{i}\right)$ the instants at which the jumps of $u$ exceed in magnitude the accepted incertainty. This is not unrealistic in the present problem, since the discontinuities of $u$ can only occur at points of discontinuity of the given multifunction $t \rightarrow C(t)$. In view of (7.3), every jump of $C(t)$, expressed in terms of Hausdorff distance, exceeds the corresponding jump of $u(t)$. One proves in fact a property of uni- 
form convergence relative to the set of the finite subsets of $I$, such as $\left(t_{i}\right)$ above, partially ordered by inclusion, i.e. the uniform convergence of some directed net of step-functions. 21

But, when tackling the approximation of discontinuous processes, another sort of convergence seems more promising : instead of measuring the distance between $u$ and $\hat{u}$ by the uniform norm, one considers the Hausdorff distance between their graphs. This amounts to take into account jointly some uncertainty about the values of the investigated functions of $t$ and some uncertainty about the values of $t$ at which they are computed. In that context, a practical convergence theorem, relative to uniform refinements of the discretization, is established. ${ }^{31}$ 


\section{A SYNTHETIC FORMULATION}

Let us come back now to the problem formulated in sect. 3 . In the general expression of the kinetic energy

$$
T(q, \dot{q})=\frac{1}{2} \underset{i, j}{\sum_{i j}} a_{q}(q) \dot{q}^{i} \dot{q}^{j},
$$

the functions $a_{i j}(q)=a_{j i}(q)$ are assumed smooth enough for the Lagrange equations to make sense. Actually the dependence of $a_{i j}$ on $q$ would in the sequel cause only technical complication, so for the sake of simplicity we shall restrict ourselves to the case where some parametrization of 2 may be found, such that these $n(n+1) / 2$ functions are constants. This amounts to say that the Riemannian metric constructed on $Q$ by putting

$$
d s^{2}=\sum_{i, j} a_{i j}(q) d q^{i} d q^{j}
$$

turns out to be locally Euclidean; in other words, we let aside the possible effect of Riemann curvature.

Equivalently $Q$ may, at least locally, be identified with an open subset of some Euclidean linear space $E$, with dimension $n$. In view of this Euclidean structure, the tangent space $E(q)$ at every point is 
identified with $E$ itself, as well as the cotangent space $E^{\prime}(q)$. The expression ( 8.1 ) of the kinetic energy now reduces to

$$
\mathrm{T}(\mathrm{q}, \dot{\mathrm{q}})=\frac{1}{2}\|\dot{\mathrm{q}}\|^{2}
$$

and the Lagrange equation (3.1), for every interval of smooth motion, becomes

$$
\ddot{q}=Q(t, q)+R \text {. }
$$

Using (2.4) as before in order to eliminate $R$ we obtain

$$
Q(t, q)-\ddot{q} \in N(q) \text {. }
$$

As in Sect. 3, for every interval of smooth motion, this is found equivalent to the stronger differential inclusion

$$
Q(t, q)-\ddot{q} \in \partial \psi(V(q), \dot{q})
$$

Let us formulate now an evolution problem involving differential measures in the way explained in Sect. 6 and 7 ; here again $I$ is a real interval, open on the right or unbounded, but containing $t_{0}$ as origin. PROBLEM $\mathrm{P}$. Given $\mathrm{q}_{\mathrm{o}} \in \mathrm{L}$ and $\dot{\mathrm{q}}_{\mathrm{o}} \in \mathrm{V}\left(\mathrm{q}_{\mathrm{o}}\right)$, to find $\mathrm{u} \in \operatorname{rclbv}(\mathrm{I}, \mathrm{E})$, with $u\left(t_{0}\right)=\dot{q}_{o}$, such that by putting 


$$
q(t)=q_{0}+\int_{t_{0}}^{t} u(\tau) d \tau
$$

one has

$$
Q(t, q(t)) d t-d u \in \partial \psi(V(q(t)), u(t))
$$

in the following sense : there exists (non uniquely) a nonnegative real measure $d \mu$ on I relatively to which the Lebesgue measure $d t$ and the differential measure $d u$ of $u$ admit the respective densities $t^{\prime}{ }_{\mu} \in \mathcal{L}_{1 \mathrm{loc}}^{1}(\mathrm{I}, \mathrm{d} \mu ; \mathrm{R})$ and $\mathrm{u}_{\mu}^{\prime} \in \mathcal{L}_{1 \mathrm{oc}}^{1}(\mathrm{I}, \mathrm{d} \mu ; \mathrm{E})$ verifying for every $t \in I$

$$
Q(t, q(t)) t^{\prime}{ }_{\mu}(t)-u^{\prime}{ }_{\mu}(t) \in \partial \psi(V(q(t), u(t))
$$

In the same way as in Sect. 7 for the sweeping process, the above formulation is found to be indifferent to changing the "base" measure du A possible solution $u$ of this Problem is locally absolutely continuous over some open subinterval $I^{\prime}$ of $I$ if and only if there exists $\dot{\mathrm{u}} \in \mathcal{L}_{1 \mathrm{oc}}^{1}\left(\mathrm{I}^{\prime}, \mathrm{dt} ; \mathrm{E}\right)$ such that, in restriction to $I^{\prime}$, one has $\mathrm{du}=\dot{\mathrm{u}} \mathrm{dt}$; then the following equalities hold between the restrictions to $I^{\prime}$ of the considered measures

$$
\mathrm{u}_{\mu}^{\prime} \mathrm{d} \mu=\dot{\mathrm{u}} \mathrm{dt}=\dot{\mathrm{u}} \mathrm{t}_{\mu}^{\prime} \mathrm{d} \mu
$$

Consequently $u_{\mu}^{\prime}=\dot{u} t_{\mu}^{\prime}$ throughout $I^{\prime}$, with the possible exception of a du-negligible, hence dt-negligible, subset. On the other hand the 
function $t^{\prime}{ }_{\mu}$ is nonnegative and the subset in which it vanishes is dtnegligible. Therefore (8.8) implies that

$$
Q(t, q(t))-\dot{u}(t) \in \partial \psi(V(q(t), u(t))
$$

holds Lebesgue-a.e. in $I^{\prime}$. This amounts to say that the function $t \rightarrow q(t)$ satisfies over $I^{\prime}$ the dynamical condition (8.5) of shockless motion.

Suppose on the other hand that a solution $u$ of Problem $P$ is discontinuous at time $t_{s} \in I$. Then the vector measure du presents at this point an atom with value $u\left(t_{s}\right)-u^{-}\left(t_{s}\right)$; equivalently the real measure $d \mu$ presents an atom with mass $\mu_{s}>0$ and

$$
u^{\prime}{ }_{\mu}\left(t_{s}\right) \mu_{s}=u\left(t_{s}\right)-u^{-}\left(t_{s}\right)
$$

Since the Lebesgue measure $d t$ has no atom, one obtains $t^{\prime}{ }_{\mu}\left(t_{s}\right)=0$ and finally (8.8) implies

$$
u^{-}\left(t_{s}\right)-u\left(t_{s}\right) \in \partial \psi\left(V\left(q\left(t_{s}\right)\right), u\left(t_{s}\right)\right)
$$

equivalent to

$$
u\left(t_{s}\right)=\operatorname{prox}\left(V\left(q\left(t_{s}\right)\right), u^{-}\left(t_{s}\right)\right)
$$

In view of the right-continuity of $u$, this is nothing else than the 
definition property (5.1) of a standard inelastic shock ; in fact (8.6) elementarily implies that the right and left limits of $u$ respectively equal the right and left derivatives of $q$.

Conversely, one checks in a similar way that a motion consisting of a sequence of intervals of smooth motion, connected by standard inelastic shocks, is a solution of Problem P. But it cannot be expected a priori that every solution of Problem $P$ exhibits such a simple structure, i.e. is of finite sort, according to the terminology of Sect. 1 .

Let us establish now a power balance formula which generalizes both relations (3.8) and (5.3). For every $t \in I$, condition (8.8) expresses that $u(t)$ and the right-hand member form a pair of conjugate points relative to the pair of Fenchel conjugate functions equal to the respective indicators of $\mathrm{V}(\mathrm{q}(\mathrm{t}))$ and $\mathrm{N}(\mathrm{q}(\mathrm{t}))$; therefore these elements are or thogonal, i.e.

$$
\forall t \in I: u(t) \cdot\left[Q(t, q(t)) t^{\prime}{ }_{\mu}(t)-u^{\prime}{ }_{\mu}(t)\right]=0
$$

As an element of $\operatorname{lbv}(\mathrm{I}, \mathrm{E})$, the function $\mathrm{u}$ is locally bounded and universally measurable; hence the above expression constitutes the density, relative to $\mathrm{d} \mu$, of some real measure. Using the notations of Sect. 6 this yields the equality of real measures

$$
u \cdot d u=u(t) \cdot Q(t, q(t)) d t
$$

Therefrom we shall derive some inequalities, emphasizing in general 
the dissipative character of the mechanical process under study.

Let us call the speed of the system the real function

$$
\sigma(t)=\|u(t)\|,
$$

belonging to $1 b v(I, \mathbb{R})$, since the norm mapping is Lipschitz from $E$ into $\mathbb{R}$. Clearly $\sigma^{+}(t)=\left\|^{+}(t)\right\|=\sigma(t)$ in view of the rightcontinuity, and $\sigma^{-}(t)=\left\|u^{-}(t)\right\|$. Observe that (8.10), established for every discontinuity point $t_{s}$, is also trivially true at continuity points since $\mathrm{u}^{-}=\mathrm{u} \in \mathrm{V}(\mathrm{q})$ at such points. As the mapping "prox" is nonexpanding, it comes out that

$$
\forall t \quad: \quad \sigma(t) \leqslant \sigma^{-}(t)
$$

Applying (6.6) successively with $X=\mathbb{R}$ and $X=E$ one obtains, in view of $\mathrm{u}^{+} \mathrm{u}^{+}$and using (8.11) and (8.12),

$$
\sigma^{-} \mathrm{d} \sigma \leqslant \frac{1}{2} \mathrm{~d}\left(\sigma^{2}\right)=\frac{1}{2} \mathrm{~d}\|\mathrm{u}\|^{2} \leqslant \mathrm{u} \cdot \mathrm{du} \leqslant \sigma\|\mathrm{Q}\| \mathrm{dt} \leqslant \sigma^{-}\|\mathrm{Q}\| \mathrm{dt} .
$$

If an upper bound $M$ of $\|Q(t, q)\|$ is available for the considered problem, this allows one to derive an upper bound of $\sigma(\tau)$, namely

$$
\sigma(\tau) \leqslant\left\|\dot{q}_{0}\right\|+\left(\tau-t_{0}\right) M
$$

In fact in the special case where $\sigma^{-}$does not vanish in the interval 
]$t_{0}, \tau[,(8.13)$ yields, for the restriction of the considered measures to this interval,

$$
\mathrm{d} \sigma \leqslant \mathrm{M} d \mathrm{t}
$$

from which (8.14) would ensue by integration and by using (6.3) and (8.12). If the subset of $] t_{0}, \tau\left[\right.$ where $\sigma^{-}$vanishes is nonempty, let us denote by $\tau_{0}$ the 1.u.b. of this subset. Every left-neighborhood of $\tau_{0}$ in $I$ contains points where $\sigma^{-}=0$, hence $\sigma^{-}\left(\tau_{0}\right)=0$ (recall that the function $t \rightarrow \sigma^{-}(t)$ is essentially left-continuous, see e.g. ${ }^{27}$ ) thus $\sigma\left(\tau_{0}\right)=0$ by (8.12). Inequality (8.15) holds for the restriction of the considered measures to $] \tau_{0}, \tau[$, then, by integration

$$
\int_{] \tau_{0}, \tau[} d \sigma=\sigma^{-}(\tau)-\sigma\left(\tau_{0}\right) \leqslant\left(\tau-\tau_{0}\right) M .
$$

Using again (8.12) one concludes

$$
\sigma(\tau) \leqslant \sigma^{-}(\tau) \leqslant\left(\tau-\tau_{0}\right) M \leqslant\left(\tau-t_{0}\right) M
$$

which establishes $(8.14)$.

We now discuss the connection of Problem $\mathrm{P}$ with the sweeping process presented in Sect. 7 .

Let us introduce the new unknown function $v \in \operatorname{rclbv}(I, E)$, $v\left(t_{0}\right)=\dot{q}_{0}, \quad b y$ 


$$
v(t)=u(t)-\int_{t_{0}}^{t} Q(\tau, q(\tau)) d \tau .
$$

In view of (8.6), the function $t \rightarrow q(t)$ is related to $v$ as being the solution of the smooth integro-differential equation

$$
\frac{d q}{d t}-\int_{t_{0}}^{t} Q(\tau, q(\tau)) d t=v(t),
$$

with initial condition $q\left(t_{0}\right)=q_{0}$. Let us express this by writing $\mathrm{q}=\mathrm{Sv}$; the nonlinear operator

$$
S: \operatorname{rclbv}(I, E) \rightarrow \mathrm{W}_{1 \mathrm{oc}}^{1, \infty}(I, E)
$$

enjoys reasonably good continuity properties. The right-hand side of (8.7) now writes down as

$$
\partial \psi(V(q(t)), u(t))=\partial \psi\left(C_{v}(t), v(t)\right),
$$

with

$$
C_{v}(t)=V(\operatorname{Sv}(t))-\int_{t_{0}}^{t} Q(\tau, \operatorname{Sv}(\tau)) d \tau
$$

Under these notations, Problem $P$ takes the equivalent form : To find $v \in \operatorname{rclbv}(I, E), v\left(t_{0}\right)=\dot{q}_{o}, \underline{\text { satisfying }}$

$$
-d v \in \partial \psi\left(C_{v}(t), v(t)\right)
$$


In other words, $\mathrm{v}$ is a solution of the sweeping process by some moving convex set $\mathrm{C}$ which itself depends on $\mathrm{v}$. Whether this allows one to prove solution existence by some fixed point argument is still an open question. But the above provides at least some insight into the structure of the problem and naturally suggests various approximation procedures.

For instance, one may apply to (8.17) the catching-up algorithm described in Sect. 7, with $\mathrm{C}_{\mathrm{v}}$ adjusted at each step through the concomitant discretization of $(8.16)$.

Another sort of approximation would proceed by "regularization" : replacing the indicator function $\psi\left(\mathrm{C}_{\mathrm{v}},.\right)$ in (8.17) by a penalty function of the set $\mathrm{C}_{\mathrm{v}}$, namely

$$
x \rightarrow \frac{1}{2 \lambda}\left[\operatorname{dist}\left(x, C_{v}\right)\right]^{2}
$$

where $\lambda$ is a "sma11" positive constant. Hence the differential inclusion (8.17) is replaced by the differential equation

$$
-\frac{d v}{d t}=\frac{1}{\lambda}[v-p r o x(C, v)]
$$

The single-valued Lipschitz mapping in the right-hand side may also be viewed as resulting from the regularization, in Yosida's style, of the monotone multifunction $\partial \psi\left(C_{v},.\right)$ (see e.g. $\left.{ }^{32,33}\right)$.

Here, as we1l as in the catching-up discretization, a certain extent of violation of the geometrical condition $q \in L$ has to be accepted, with 
some adequate definition of $\mathrm{V}(\mathrm{q})$ for $\mathrm{q} \notin \mathrm{L}$. . The regularization procedure used in ${ }^{12,13}$ consists in exerting some elastic pul1-back as soon as this condition is violated : this agrees with the concept of an energypreserving shock. In contrast, the above amounts to apply some breaking action of the viscous type, with coefficient $1 / \lambda$, as soon as the kinematical condition $\dot{\mathrm{q}}^{+} \in \mathrm{V}(\mathrm{q})$ is violated : this is consistent with the dissipative character of inelastic shocks. 


\section{REFERENCES}

1. Bressan, A., Incompatibilità dei teoremi di esistenza e di unicità del moto per un tipo molto comune e regolare di sistemi meccanici, Ann. Scuola Norm. Sup. Pisa, Ser. III, 14, 333, 1960.

2. Delassus, E., Mémoire sur la théorie des liaisons finies unilatérales, Ann. Sci. Ecole Norm. Sup., 34, 95, 1917.

3. Bouligand, G., Compléments et exercices sur la mécanique des solides, 2ème édition, Vuibert, Paris, 1945.

4. Bouligand, G., Mécanique rationnelle, 5ème édition, Vuibert, Paris, 1954 .

5. Moreau, J.J., Les liaisons unilatérales et le principe de Gauss, C.R. Acad. Sci. Paris, 256, 871, 1963.

6. Moreau, J.J., Quadratic programming in mechanics : dynamics of onesided constraints, SIAM J. Control, 4, 153, 1966 (Proceedings of the First International Congress on Programming and Control).

7. Moreau, J.J., Sur la naissance de la cavitation dans une conduite, C.R. Acad. Sci. Paris, 259, 3948, 1965.

8. Moreau, J.J., Principes extrémaux pour le problème de la naissance de 1a cavitation, J. de Mécanique, 5, 439, 1966. 
9. Moreau, J.J., One-sided constraints in hydrodynamics, in Non linear Programming, Abadie, J., Ed., North-Holland Pub. Co., Amsterdam, $1967,257$.

10. Kilmister, C.W. and Reeve, J.E., Rational mechanics, Longmans, London, 1966.

11. Moreau, J.J., Mécanique classique, Vol 2, Masson, Paris, 1971.

12. Schatzman, M., Le système différentiel $\left(\mathrm{d}^{2} \mathrm{u} / \mathrm{dt} \mathrm{t}^{2}\right)+\partial \varphi(\mathrm{u}) \ni \mathrm{f}$ avec conditions initiales, C.R. Acad. Sci. Paris, Série 1, 284, 603, 1977.

13. Schatzman, M., A class of non linear differential equations of 2 nd order in time, J. Nonlinear Analysis, Theory, Methods and Appl., 2, 355, 1978 .

14. Buttazzo, G. and Percivale, D., Sull'approssimazione del problema del rimbalzo unidimensionale, Scuola Norm. Sup. Pisa, E.T.S. Pisa, 1980.

15. Buttazzo, G. and Percivale, D., The bounce problem on n-dimensional Riemannian manifolds, Scuola Norm. Sup. Pisa, E.T.S. Pisa, 1981.

16. Amerio, L. and Prouse, G., Study of the motion of a string vibrating against an obstacle, Rend. Mat. , 8, 563, 1975.

17. Schatzman, M., A hyperbolic problem of second order with unilateral constraints : the vibrating string with a concave obstacle, J. Math. Ana1. App1., 73, 138, 1980.

18. Cabannes, H. and Haraux, A., Mouvements presque-périodiques d'une corde vibrante en présence d'un obstacle fixe, rectiligne ou ponctual, Int. J. Non-linear Mechanics, 16, 449, 1981 .

19. Do, C., On the dynamic deformation of a bar against an obstacle, in 
Variational Methods in the Mechanics of Solids, S. Nemat-Nasser, Ed., Pergamon Press, 1980 .

20. Moreau, J.J., Liaisons unilatérales sans frottement et chocs inélastiques, C.R. Acad. Sci. Paris, Série II, 296, 1473, 1983.

21. Moreau, J.J., Evolution problem associated with a moving convex set in a Hilbert space, J. Diff. Equ., 26, 347, 1977.

22. Abadie, J., On the Kuhn-Tucker theorem, in Non linear Programming, Abadie, J., Ed., North-Holland Pub. Co., Amsterdam, 1967, 17.

23. Moreau J.J., Décomposition orthogonale d'un espace hilbertien selon deux cones mutuellement polaires, C.R. Acad. Sci. Paris, 255, 238, 1962.

24. Moreau, J.J., Proximité et dualité dans un espace hilbertien, Bul1. Soc. Math. France, 93, 273, 1965.

25. Moreau, J.J., On unilateral constraints, friction and plasticity, in New variational Techniques in Mathematical Physics, G. Capriz and G. Stampacchia, Eds., CIME II Ciclo 1973, Edizioni Cremonese, Roma, $1974,173$.

26. Halphen, B. and Nguyen, Q.S., Sur les matériaux standards généralisés, J. de Mécanique 14, 39, 1975.

27. Moreau, J.J., Sur les mesures différentielles de fonctions vectorielles, in Séminaire d'Analyse Convexe, Montpellier, 5, exp. 17, 1975.

28. Moreau, J.J., Sur les mesures différentielles et certains problèmes d'évolution, C.R. Acad. Sci. Paris, Sér. A-B, 282, 837, 1976.

29. Moreau, J.J., Multiapplications à rétraction finie, Ann. Scuola Norm. 
Sup. Pisa, C1. Sci. Ser. IV, 1, 169, 1974.

30. Bourbaki, N., Intégration, Chap. 6 (E1éments de Mathématique, fasc. XXV), Hermann, Paris, 1959.

31. Moreau, J.J., Approximation en graphe d'une évolution discontinue, RAIRO Analyse Numérique, 12, 75, 1978.

32. Brezis, H., Problèmes unilatéraux, J. Math. Pures App1., 51, 1, 1972.

33. Brezis, H., Opérateurs Maximaux Monotones et Semi-groupes de Contractions dans les Espaces de Hilbert, Math. Studies, 5, North-Holland, Amsterdan, 1973. 